\title{
A note on measuring political participation in comparative research
}

\author{
JAN W. VAN DETH \\ Department of Public Administration. Twente University of Technology, P.O. Box 217. \\ 7500 A Enschede, the Netherlands
}

\begin{abstract}
This article presents an application of Prezeworski and Teune's so-called "identityequivalence method" to a large set of indicators of political participation. By relaxing commonly held assumptions about necessary distinctions among types of participation, it is found that the distinction between "conventional" and "unconventional" modes of participation is unnecessary, while the distinction between "government" and "nongovernment" has some merit. The findings also lend further support to the claims of Prezeworski and Teune that the identity-equivalence method is preferable to the identical indicator method.
\end{abstract}

\section{Introduction}

The term 'political participation' usually refers to behavior like voting, campaigning, demonstrating, rioting, and the like. These modes of participation vary widely between distinct political systems and cultures, and between different points in time. The obvious question, then, is: how to reach meaningful conclusions about the similarities and differences in both the modes and the level of participation in cross-national, cross-cultural and/or longitudinal research? Frequently, identical instruments are designed and applied for different contexts and the assumption is introduced that possible variations among subgroups are 'real', i.e. not due to differences in the meaning that identical measures can have for these distinct groups.

This solution is hardly acceptable in the case of political participation. The german concept 'Bürgerinitiative', for instance, has much in common with what is called 'inspraak" in dutch. Both terms refer to more or less new and non-electoral modes of participation. But the concepts are certainly not identical. Since there is no literal counterpart for the german 'Bürgerinitiative' in dutch, a comparison of the levels of participation in these countries cannot be undertaken when literal translations of the instruments are used. So, we either drop all references to nation or time specific modes of participation, or we try to establish equivalent instead of identical instruments.

* Parts of this article have been included in a paper presented at the Joint Sessions of the European Consortium for Political Research, workshop on 'Political participation in advanced industrial societies', Florence, March 1980. 
The prospects of the last option are evaluated in this article by analyzing data on political participation in eight advanced industrial countries (USA, Britain, West Germany, Italy, the Netherlands, Austria, Finland, and Switzerland). ${ }^{1}$ Both the modes and the level of participation in these countries will be compared by developing equivalent instruments for this type of behavior. No a priori distinctions between, say, conventional and unconventional modes of participation are accepted and the focus is not limited to actions specifically addressed to the governments. This search for equivalent measures will lead to a challenge of long-held assumptions concerning necessary distinctions among types of participation.

\section{The many faces of participation}

Research on participation is a major branch of political science. Conflicts between proponents of the instrumentalist, the developmental, and the system-oriented views have dominated the normative part of the field, while a virtual endless number of conceptualizations, operationalizations, schemes, typologies, taxonomies, aspects, dimensions or factors can be found in the more empirical approaches. ${ }^{2}$ I am neither concerned with normative theory nor with yet another a priori classification of distinct modes of participation. What is needed here is a demarcation line between political participation and the rest of human behavior as a point of departure for the construction of a cross-national instrument. Two common distinctions will be considered: the one between governmental and nongovernmental oriented behavior, and the one between conventional and unconventional modes of participation.

Let us start with a minimum definition of political participation as "taking part' in politics. Consequently, the well-known Eastonian definition can be used to describe politics as '...those interactions through which values are authoratively allocated for a society' (Easton, 1965:21). Skipping over the debates on this definition, the concept will be summarized here as the process of creation and allocation of values. From this it follows that political participation includes those types of behavior which enable the citizen(s) to take part in the process of creation and allocation of values for a society. ${ }^{3}$

Two quite distinet remarks can be made in the side line. First, the behavior will be intentional most of the time - i.e. meant to influence the process - but does not have to be so. For instance, working for a candidate can be motivated by the desire to improve career opportunities, without the intention to influence the process of creation and allocation of values for a society. However, the behavior remains politically relevant and therefore an act of participation. Second, 'voluntariness' can also be left out as a distinct feature of political participation. Only rather strange definitions of voluntary activities can bring, for instance, protest against a planned highway through your yard under that concept. Besides, the behavior remains, politically relevant whether it is voluntary or not. 
In many countries, both the creation and allocation of values are not monopolies of government agencies, and so participation can be found in other areas of these societies as well. Participation is the link between the needs and interests of the individual and the process of creation and allocation of values. Na additional theoretical arguments are required to justify a conceptualization of participation in a broader, not specifically governmental oriented context. On the contrary, arguments are needed to restrict a definition like that. A broad concept of politics has clear advantages from both an analytical and a comparative point of view. Limiting the focus to the government sector provides problems because the value scope of that sector varies with time and/or places (see Helmers et al., 1975: 25-30).

Furthermore, it is doubtful whether people are always able to differentiate their needs and interests according to some governmental-nongovernmental criterion. Even in nonauthoritarian or nontotalitarian regimes, it is often hard to tell what exactly belongs to the government sector and what are purely private creations and allocations of values. Governments of advanced industrial societies have expanded their activities since 1945, providing their citizens with guarantees for their basic needs, and with regulations for many of their activities. In turn, citizens present even more demands to their governments and expect 'official' attention and help for many of the problems they face. Especially a liberal democracy seems to generate excessive demands and expectations, and a demarcation line between typical governmental activities and nongovernmental tasks becomes more and more problematical. On the micro-level the individual is confronted with an environment intervening ever deeper in his affairs, irrespective whether it concerns his role as a worker loosing his job, a consument watching television, or a citizen in need of a building license, a subsidy for his soccer club or a welfare worker for his marriage problems. So the question whether or not political participation should be restricted to the governmental sector cannot be answered at forehand and should be left to the (potential) participants (cl. Hirschman, 1982).

An analogical argument holds for the distinction between conventional and unconventional modes of participation. Many labels have been proposed to identify the types of behavior that should belong to the last category. Beside the rather neutral terms 'protest' and 'political action' there are adjectives like untraditional, direct, nonelectoral, not legitimate, not legal, noninstitutionalized, unorthodox, and - as mentioned - unconventional. Many of these definitions are status-quo oriented and have a negative surplus meaning.

The most interesting attempt to conceptualize this specific type of political participation can be found in the work of Alan Marsh. He developed instru. ments to measure, what he calls, 'unconventional political behavior'. Respondents were confronted with a set of ten stimuli: petitions, demonstrations, boycotts, refusing rents and taxes, strikes, painting slogans, occupations, blockades, damaging property, and violence. The affective, conative, and cognitive components of the attitudes towards these modes of participation could be stated by most of the respondents. Applying the deterministic 
cumulative model of Louis Guttman, Marsh was able to construct a 'protest potential scale' and a typology of political activists (Marsh, 1974; 1977; 1979; Marsh and Kaase, 1979).

Does this sophisticated approach to the protest phenomenon implies that there is a clear distinction between conventional and unconventional modes of participation? I'm afraid not. Marsh has demonstrated what he intented to do: that there is constraint within his set of items for unconventional political behavior. The fact that these attitudes can be measured does not, of course, imply that conventional and unconventional modes of participation are disjunct categories.

In summary, the arguments for a priori distinctions between different modes of participation are not convincing, and do not lead to restrictions on the broad, non-specific definition of politics presented above. There is no reason to expect that the average citizen is inclined to distinguish sharply between governmental and nongovernmental oriented modes of participation, or between conventional and unconventional types of political behavior. To test this proposition, the constraints within a large and heterogenious set of items to measure political behavior should be investigated.

\section{Strategy and model}

In the eight advanced industrial countries under consideration, several stimuli have been used to measure distinct modes of political participation. I will pool these items in order to search for a unidimensional representation that is in line with the broad conceptualization of politics. As an initial set of items for the construction of a participation scale, nineteen items were selected. These are: seven items for 'conventional participation' (read about politics, discuss politics, convince friends, solve local problems, attend a meeting, contact officials, and working for a candidate), the ten items of Marsh for 'unconventional participation' mentioned above, and the membership of a political party or a union. Further enlargement of the set was not possible due to missing items for some of the countries.

The assumption that the conative aspects of the participation concept form a single dimension - from commonplace to extreme - can be tested with the model of Guttman. That is what Marsh did when he thought of the basic question underlying his stimuli: 'Generally speaking, how far are you prepared to go?' (Marsh, 1977: 48). The Guttman model, however, is very vulnerable to inflation of the scale coefficients and lacks a statistical theory and statistical tests to interprete possible response errors or possible differences of the coefficients among distinct subgroups.

As an alternative of the deterministic model, the cumulative scale analysis procedure proposed by Robert Mokken will be used. The basic idea underlying this approach is identical to the Guttman model. Instead of the deterministic assumptions, it is assumed that each subject has a certain, unknown value 
on some latent dimension. For each item, the probability of a positive response increases monotonically with that unknown value. Distinct scale coefficients of scalability can be derived from the correlations between the items: $H(i j)$ for each pair of item $i$ and item $j, H$ for the complete scale, and $H(i)$ for each item $i$ with respect to the rest of the scale.

For the construction of stochastic scales from a pool of items, a stepwise procedure can be applied. Starting with either a defined set of items or the pair with the highest $H(i j)$-value, an item is added to the scale in every step as long as the conditions for such a scale are fulfilled (i.e.: $(H(i j)>0$, and $H>$ constant). For the remaining items, a search for a next scale is started. And so on. (Mokken, 1970; see for a concise overview of the model Stokman and Van Schuur, 1980; or Niemöller and Van Schuur, 1983).

The introduction of the Mokken model still leaves us with the problems of translation and equivalence of instruments in comparative research. Do we have to use identical sets of items for each country as the authors of "The Civic Culture' and 'Political Action' believe? Or is there a way to construct equivalent, nation-specific scales that takes into account the particularities of the political cultures of the eight countries? Twenty years ago, Prezeworski and Teune suggested an approach to comparative research along the lines implied by the last alternative. Their so-called 'identity-equivalence procedure' begins with the selection of a set of intercorrelated items from a pooled cross-country analysis of a large number of items. This subset of selected indicators is assumed to have cross-national validity for some concept and the countries considered. In other words, the items form a scale regardless of cultural differences. The set of selected indicators is called the 'identity set'. Then, all items are analyzed for each country separately in order to test the identity set and to select items which are correlated with this set in some nation-specific way. The result can be that - apart of the common indentity set - different scales are found for each country. This provides the opportunity to create longer, and thus more valid scales. The cross-national equivalence of these non-identical scales is based on the use of the identity set (Prezeworski and Teune, 1966).

The 'identity-equivalence procedure' seems to be very useful for an exploration of the cross-national differences of the troublesome concept 'political participation'. If we have found an identity set and added different items for different countries, the following three types of comparisons can be made with this procedure:

1. comparison of the modes of the latent variable, i.e. the different items added to the identity set for nation-specific modes of participation;

2. comparison of the level of the variable, i.e. the level of participation as measured by the scores on the distinct scales;

3. comparison of the constraint of the elements, i.e. the degree of constraint between the modes of participation as measured by the coefficients of scalability.

Type one and type two can be used for comparative research; type three is 
only applied at that level when it has to be shown that the items of the identity set are related in similar ways in distinct countries. However, each type presupposes the existence of an identity set for the concept under consideration. The first task, then, is the construction of such a set for the measurement of political participation in the eight advanced industrial countries.

\section{Constructing equivalent scales}

Analyzing the pooled eight country data, a remarkable scale with as much as 14 of the initial 19 items resulted from the search procedure. ${ }^{4}$ This scale consists of all items except 'refusing rents and taxes', 'strikes', 'damaging proptery', 'violence', and 'ution membership'.

The excluded items form two distinct, two-item scales: one for 'strikes' and 'union membership' ( $H=0.52$; a strong scale), and another for 'damaging property' and 'violence' ( $H=0.35$; a weak scale). This spread over two additional scales is rather trivial since both industrial actions and the use of violence can be seen as special types of participation. However, these results are certainly not in line with the broad concept of politics presented above. I will return to this point in the last paragraph.

The item 'refusing rents and taxes' is not scalable with any subset of the other items in the pool. The direct and individual profits that can be obtained with this type of action probably disturb the motivation to use it as a mode of political participation among other modes of participation.

The exclusion of these particular five items from the final scale seems plausible. In Table 1 it can be seen that the remaining 14 items form a clear cumulative scale ( $H=0.48$; a medium scale).

All item coefficients in Table 1 are above the generally accepted lowerbound of 0.30 . But the scale does not meet another requirement for the meaningful application of the Mokken model. As a stochastic model double monotone or holomorphic sets of item are assumed. Unfortunately, this assumption is violated by many of the items in he scale. ${ }^{5}$ First of all, the items 'petitions', 'painting slogans', 'occupations' 'blocking traffic', and 'working for a candidate' must be deleted from the scale because of very clear violations. Second, the scalability of the items 'convince friends', and 'membership of a political party' is dubious. Since the frequency of this last item is almost identical to that of 'demonstrations', and 'convince friends' is about as difficult as 'contact officials', these two ambiguous items can be deleted too.

This reconsideration of the result of the search procedure for the pooled data set, leaves us with a 7 -items scale as the cross-national identity set ( $H=0.57$; a strong scale). Furthermore, the constraints among these items are similar in each country. This can be lested with Mokken's statistical procedure for possible differences of the scale coefficients for distinct subgroups (the countries). For none of the eight countries under consideration the null 
Table 1. Selected items and scale coefficients (Mokken scale; pooled set of eight countries)

\begin{tabular}{lll}
\hline item & difficulty & $H(i)$ \\
\hline read politics & 0.83 & 0.68 \\
discuss politics & 0.75 & 0.71 \\
solve local problems & 0.38 & 0.47 \\
altend meetings & 0.35 & 0.51 \\
contact officials & 0.31 & 0.49 \\
try to convince friends & 0.31 & 0.42 \\
signing a petition & 0.30 & 0.32 \\
working for a candidate & 0.16 & 0.56 \\
demonstrations & 0.09 & 0.41 \\
member political party & 0.09 & 0.42 \\
boycotls & 0.05 & 0.39 \\
blocking traffic & 0.01 & 0.45 \\
occupations & 0.01 & 0.40 \\
painting slogans & 0.01 & 0.46
\end{tabular}

scale coefficient $H=0.48 ;$ rho $=0.81$

hypothesis of equal $H$ and $H(i)$ across the nations had to be rejected. ${ }^{6}$ The scale properties of the resulting identity set are shown in Table 2.

Having constructed an identity set for the eight nations, the next step is the search for country-specific additions from the remaining items to measure political participation. For each country considerable extensions of the identity set can be found, but - just as with the identity set - most of these items have to be deleted after checking the holomorphism of the final item sets.

In spite of these complications, several items meet the criteria for addition to the scales. The item 'petitioning' can be included in the scales for Britain, Germany, the Netherlands, and Finland. For Italy and Switzerland, the scales can be extended with some other 'unconventional' items, but these items are not added because they are extremely difficult and the scales already contain a very difficult item of this type. The items 'convince friends' and 'working for a candidate' can be used to improve the instruments for the Netherlands, the

Table 2. Cross-national identity set (Mokken scale; pooled set of eight countries)

\begin{tabular}{lll}
\hline item & difficulty & $H(i)$ \\
\hline read politics & 0.83 & 0.57 \\
discuss politics & 0.75 & 0.69 \\
solve local problems & 0.38 & 0.52 \\
attend meetings & 0.35 & 0.54 \\
contact of ficials & 0.31 & 0.57 \\
demonstrations & 0.09 & 0.49 \\
boycotts & 0.05 & 0.39 \\
\hline
\end{tabular}

scale coefficient $H=0.57$; rho $=0.77$ 


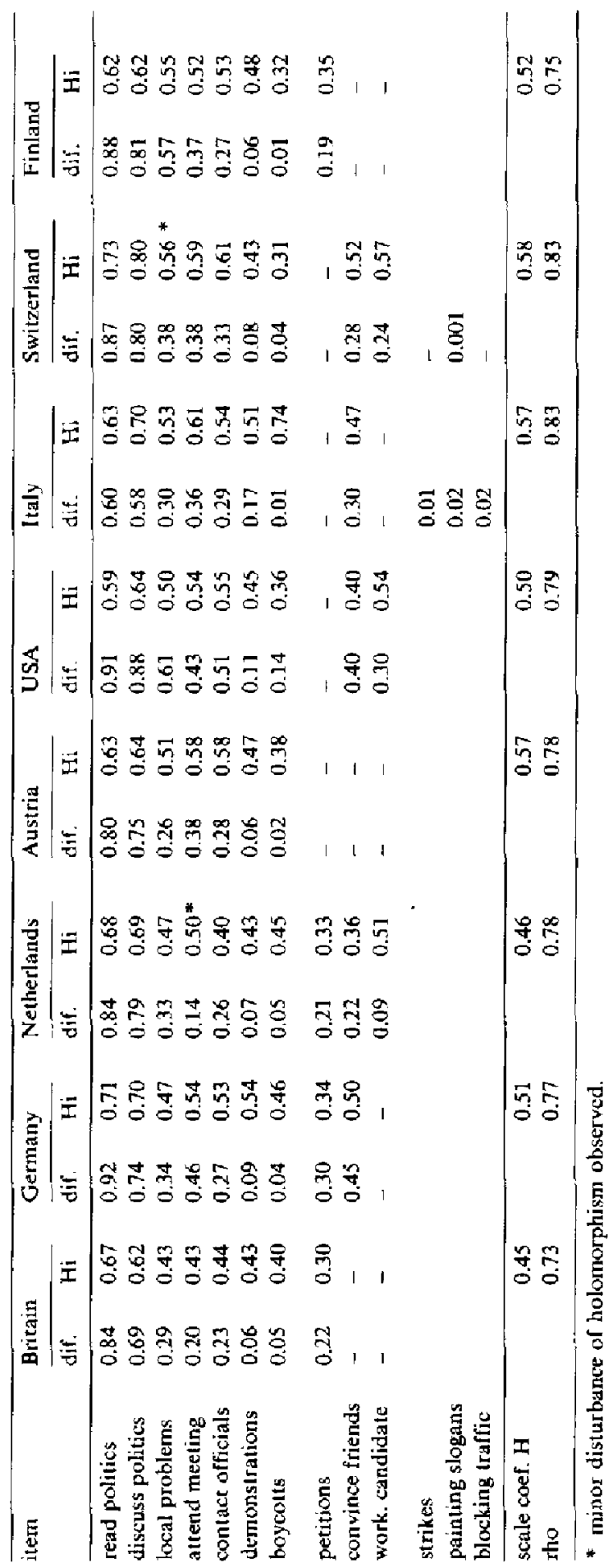


USA, and Switzerland. For Germany and Italy only 'convince friends' appears to be a useful extension of the scale.

In Table 3 the results are shown of the search for nation-specific additions to the identity set. Except for Austria, extensions can be realized for every country. These additions do not lead to violations of the assumption of holomorphism, and the coefficients of the final scale are quite satisfactory. Furthermore, both the most difficult and the easiest item in each country remain part of the identity set; all extensions are nation-specific fillings of the gaps in the continuum. The only thing left to be desired are more equally spaced items in some regions. Especially the space between 'discuss politics' and 'solve local problems' should be filled. However, the data set does not include items to be used in that way.

The search procedures for each country separately resulted in some other scales. For the Netherlands, the USA, and Finland, two-item scales can be found for 'strikes' and 'union membership'. Since the pooled country analysis also produced this scale, there will be respondents in the other five countries with similar response patterns. The inevitable conclusion is that the industrial items cannot be considered as parts of a single political participation continuum. Before I turn to the consequences of this finding, the usefulness of the approach described in this paragraph will be illustrated by comparing the levels of participation in the eight countries under consideration.

\section{Comparing the levels of participation}

Following the distinct phases in the process of scale construction as presented in the previous paragraph, the similarities and differences among the countries in both the modes of participation and the constraints among the items has become clear. Now, we can use these nation-specific scales as equivalent instruments to measure the levels of participation in these countries.

Scoring objects on a cumulative scale can be done by counting the number of positive responses for each respondent. In this case, however, the simple scores have to be corrected for the different lengths of the nation-specific scales. For instance Austrian respondents have only 7 chances to score on their scale, while the Dutch have 10 opportunities. This can be corrected by dividing the score of each respondent by the length of the scale for his country. In that way, all scores are standardized for the interval between 0 (no participation) and 1 (maximum level of participation).

The frequency distributions of the levels of participation in the eight countries are shown in Fig. 1. The points in that illustration have been connected with straight lines to facilitate visual inspection.

Except for Italy, the curves in Fig. 1 are single peaked with tops in the range between 0.2 and 0.4 . In spite of this similarity, the scales clearly discriminate between the levels of participation in the distinct countries. Remarkable is the high proportion Italians not participating at all (more than 


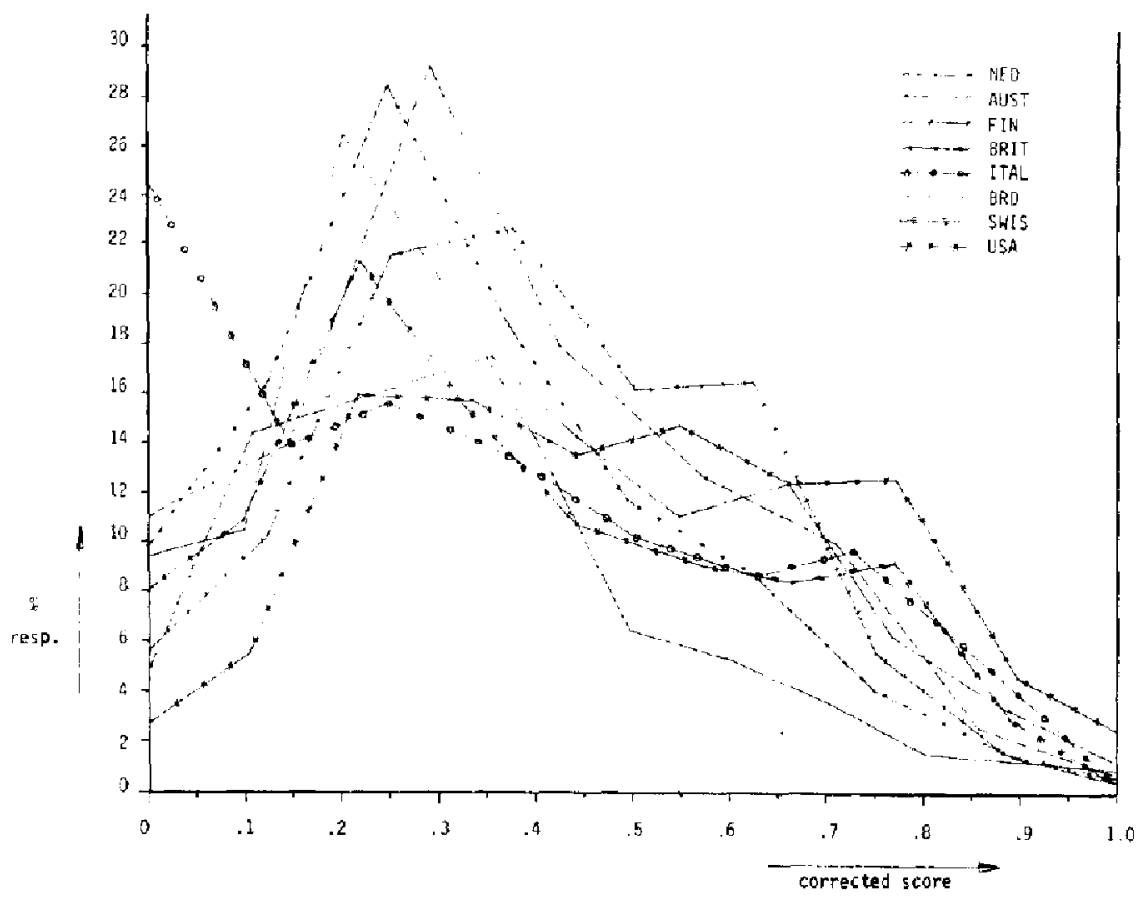

Fig. I. Distributions of the level of participation in eight countries (in percentages).

twice as much as in any other country), and the low number of participants in the Netherlands as higher levels of participation are considered. More equally distributed patterns are obtained for both the USA and Germany.

A detailed analysis of the cross-national differences in the levels of participation in the eight countries is outside the scope of this note. Fig. 1 illustrates that meaningful results can be obtained with the scales. Therefore, comparative research on political participation should not be frustrated by the common-sense view or folk-wisdom that using identical instruments is the preferred way to measure the same concept in distinct settings.

\section{Conclusions}

Starting with a broad conceptualization of politics and political participation, this exploration seems fruitful from both a theoretical and a methodological point of view.

The construction of unidimensional cumulative scales to measure the conative component of the attitudes towards political participation provides support for the idea that it is superfluous to make an a priori distinction between 'conventional' and 'unconventional' modes of participation. On the contrary, both types can be mixed in unidimensional scales. These scales have 
good technical proporties. However, the second assumption - politics not restricted to the governmental sector - has to be modified. The industrial items in the set do not scale with any of the other items, but form a distinct two-item scale in the pooled country analysis as well as in several countries separately. Apparently, industrial action does not fit in a broad, unidimensional conceptionalization of political participation in advanced industrial countries.

From a methodological point of view, the use of the 'identity-equivalence procedure' of Prezeworski and Teune proved to be very promising for comparative research. After the construction of the identity set, participation in the distinct countries can be assessed and compared in three ways. First, it is possible to look at the nation-specific modes of participation; second, the constraints among the modes of participation can be compared; and third, the levels of participation can be computed for each country separately. The illustration presented in this note makes clear that the construction of equivalent instead of identical instruments can provide meaningful information for each of these three aspects of comparative research on political participation.

\section{Notes}

1. These data are part of the so-called 'Political Action Project'. The total number of respondents is 12,588; questioned in 1974/75. Data were made available by the Zentralarchiv für Empirische Sozialforschung, Universität zu Köln, West Germany (study number 0765). Ncither the Archiv nor the principal investigators are responsible for the analyses presented in this article.

2. For a concise overview of the literature see Milbrath and Goel (1977).

3. This definition is a modification of the definition presented by Van der Lijk (1975).

4. All computations are performed with the stand-alone versions of the "Mokken-scale' and "Mokken-test" algorithms of the University of Amsterdam. I wish to thank Bas Tummers for rendering assistance with the conversion of the programs to the computer of Twente Tech.

5. The violations of this assumption can be established with the so-called $p(j j)$-matrix. This matrix consists of the estimates of the probabilities that two items both have positive responses. If holomorphism exists, rows and columns should increase monotonically. To have a measure for deviations from this pattern, a binominal distribution of the estimates in the $p(i i)$-matrix is assumed. All differences within a $95 \%$ interval are neglected; the remaining are 1reated by deleting items from the ser. I am indebted to Wijbrandt van Schuur for suggesting this procedure to me.

6. A so-called Mokken- $T$ statistic has been computed: $T=11.65$; significance $=0.1125$ (chisquare distribution with 7 degrees of freedom).

\section{References}

Barnes, S. and Kaase, M., et al. (1979). Political action, Mass participation in five western democracies. Beverly Hills, London: Sage.

Easton, D. (1965). A system analysis of political life. New York: Wiley.

Helmers, H.M., Mokken, R.J., Plijter, R.C., Stokman, F.N. (1975). Graven naar macht, op zoek naar de kern wan de Nederlandse economie. Amsterdam: Van Gennep.

Hirsthman, A.O. (1982). Shifting involvements, Private interest and public action. Princeton: Princeton U.P. 
Marsh, A. (1974). Explorations in unorthodox political behavior: a scale to measure protest potential. European Journal of Political Research 2:107-29.

Marsh, A. (1977). Protest and political consciousness. Beverly Hills, London: Sage.

Marsh, A. (1979). The matrix of political action. Futures 11 (2): 91-103.

Marsh, A. and Kaase, M. (1979). Political action, pp 27-103 in Barnes, Kaase et al. (1979).

Milbrath, L.W. and Goel, M,L. (1977). Political Participation. Chicago: Rand.

Niemöller, B. and Van Schuur, W.H. (1983). Stochastic models for unidimensional scaling, pp 120-70 in D. McKay, Schofield, N. and Whiteley, P. (eds), Data analysis and the sacial sciences. London: Pinter.

Prezeworski A. and Teune, H, (1966). Equivalence in cross national research. Public Opinion Quarterly 30: S\$1-68.

Stokman, F. and Van Schuur, W.H. (1980). Basic scaling, Quality and Quantity 14: 5-30.

Van der Eijk, C. (1975). Politieke participatie. Acta Politica 10: 341-63. 